\title{
Generation of experimental allergic airways inflammation in the absence of draining lymph nodes
}

\author{
Beata U. Gajewska, ${ }^{1}$ David Alvarez, ${ }^{1}$ Mariana Vidric, ${ }^{1}$ Susanna Goncharova, ${ }^{1}$ \\ Martin R. Stämpfli, ${ }^{1}$ Anthony J. Coyle, ${ }^{2}$ José-Carlos Gutierrez-Ramos, ${ }^{2}$ \\ and Manel Jordana ${ }^{1}$
}

${ }^{1}$ Department of Pathology and Molecular Medicine, and Division of Respiratory Diseases and Allergy, Centre for Gene
Therapeutics, McMaster University, Hamilton, Ontario, Canada
${ }^{2}$ Millennium Pharmaceuticals Inc., Cambridge, Massachusetts, USA

Address correspondence to: Manel Jordana, Health Sciences Centre Room 4H17, Department of Pathology and Molecular Medicine, Centre for Gene Therapeutics, McMaster University, 1200 Main Street West, Hamilton, Ontario, Canada L8N $3 Z 5$. Phone: (905) 525-9140 ext. 22473; Fax: (905) 522-6750; E-mail: jordanam@mcmaster.ca.

Beata U. Gajewska and David Alvarez contributed equally to this work.

Received for publication February 27, 2001, and accepted in revised form May 22, 2001.

\begin{abstract}
The objective of this study was to investigate the contribution of secondary lymphoid organs in the generation and maintenance of experimental allergic airway inflammation. We employed a previously reported murine model of respiratory mucosal allergic sensitization, induced by repeated aerosolizations of ovalbumin in the context of a GM-CSF airway environment. We executed this protocol in wildtype (WT) and lymphotoxin- $\alpha$-deficient mice (LT $\alpha-\mathrm{KO}$ ) mice, which are devoid of lymph nodes (LNs) and possess rudimentary spleen structures. Despite the lack of pulmonary LNs draining the airway compartment, $\mathrm{LT} \alpha-\mathrm{KO}$ mice were fully capable of mounting a robust inflammatory response in the airways, consisting of Th2 polarized $\mathrm{CD}^{+} \mathrm{T}$ cells and eosinophils. This was accompanied by IL-5, IL-13, and IFN- $\gamma$ production by splenocytes and generation of ovalbumin-specific serum IgE. Exposure to the same antigen 7 weeks after complete resolution of airway inflammation once again induced a Th2 polarized infiltrate, demonstrating intact immunological memory. To investigate inherent plasticity in establishing antigen-specific immunity, mice were splenectomized before sensitization. Allergic sensitization was completely abrogated in splenectomized LT $\alpha-\mathrm{KO}$ mice, compared with eusplenic LT $\alpha$-KO controls. These data demonstrate that secondary lymphoid organs, either LN or spleen, are essential for the generation of allergic airway responses.
\end{abstract}

J. Clin. Invest. 108:577-583 (2001). DOI:10.1172/JCI200112627.

\section{Introduction}

Current understanding is that antigens that penetrate the mucosae are taken up by antigen presenting cells (APCs), mainly dendritic cells (DCs), and transported to the regional lymph nodes (LNs). In the LNs, these antigens are presented in the context of MHC molecules to naive $T$ cells that will, then, undergo differentiation (1). An extension of this notion with reference to allergic asthma would assume that allergen-specific $\mathrm{T}$ cells are generated in the LN compartment, undergo Th2 differentiation, and traffic to the airway, where they execute their effector function including, most notably, production of a distinct cytokine and chemokine program that results in eosinophilic inflammation, goblet cell hyperplasia, and bronchial hyperreactivity (2-4). Evidence that intranasal delivery of antigen leads to activation events in the cervical LNs $(5,6)$, and a recent elegant demonstration of DC migration from the lung to the thoracic LNs in response to allergen aerosolization (7), support a role of the regional LNs in the generation of immune responses to aeroallergens. Yet the functional requirement of the thoracic LNs in the generation of allergic responses has not been directly investigated.
Lymphotoxin- $\alpha$-deficient mice (LT $\alpha-\mathrm{KO})$ are born without morphologically detectable LNs or Peyer's patches; in addition, they have an altered splenic architecture, with the loss of the typical $\mathrm{T}$ and $\mathrm{B}$ cell areas and deficient germinal center formation (8-10). Hence, LT $\alpha$-KO mice provide a particularly suitable model to investigate the role of secondary lymphoid structures in the generation of mucosal immune responses. To investigate the role of LNs in this process, we used a murine model in which allergic sensitization is achieved by repeated aerosolizations of ovalbumin (OVA) in the context of a GM-CSF airway environment, which is established by gene transfer of a replicationdeficient adenovirus encoding murine GM-CSF (11).

Our data show that, despite a complete deficiency of all peripheral LNs, LT $\alpha$-KO mice subjected to this experimental protocol mount a robust airway inflammatory response that is qualitatively comparable and quantitatively greater than that observed in wild-type (WT) littermates. In vivo antigen recall responses, Th2-associated $\operatorname{IgE}$ synthesis, and in vitro cytokine production confirmed the establishment of antigen-specific immunity and memory. To investigate the site of allergic sen- 
sitization in LT $\alpha-\mathrm{KO}$ mice, we surgically removed the spleen. Splenectomy of LT $\alpha$-KO mice before the initiation of our protocol prevented sensitization and airway inflammation, whereas splenectomy of WT littermates did not affect the generation of allergic sensitization. These data establish a critical requirement of secondary lymphoid organs in the generation of Th2 responses to aeroallergens and intimate the plasticity of the immune system in the generation of mucosal immunity.

\section{Methods}

Animals. C57BL/6 and LT $\alpha-\mathrm{KO}$ mice (5-8 weeks old) were purchased from The Jackson Laboratory (Bar Harbor, Maine, USA). Mice were housed in a specific pathogen-free environment after a 12-hour light-dark cycle. All experiments performed were approved by the Animal Research Ethics Board of McMaster University. Model of respiratory mucosal allergic sensitization. Replication-deficient human type 5 adenoviral construct encoding murine GM-CSF cDNA in the $\mathrm{E} 1$ region of the viral genome (Ad/GM-CSF) was delivered intranasally 24 hours before the first OVA exposure. Ad/GM-CSF was administered intranasally at a dose of $3 \times 10^{7} \mathrm{pfu}$ in $30 \mu \mathrm{l}$ of PBS vehicle (two $15-\mu \mathrm{l}$ administrations, 5 minutes apart) into anesthetized animals. Over a period of 10 consecutive days (days $0-9)$, mice were placed in a Plexiglas chamber $(10 \mathrm{~cm} \times 15 \mathrm{~cm} \times 25$ $\mathrm{cm})$ and exposed for 20 minutes daily to aerosolized OVA (1\% $\mathrm{wt} / \mathrm{vol}$ in $0.9 \%$ saline). OVA aerosol was generated by a Bennet nebulizer (Puritan-Bennet Corp., Carlsbad, California, USA) at a flow rate of $10 \mathrm{l} / \mathrm{min}$. For in vivo rechallenge with OVA, mice were reexposed to a $1 \%$ OVA aerosol for 20 minutes daily for 3 consecutive days, after complete resolution of initial airways inflammation.

Collection and measurement of specimens. At various time points, mice were sacrificed and bronchoalveolar lavage (BAL) was performed according to standard protocol (11). Briefly, the lungs were dissected and the trachea was cannulated with a polyethylene tube (Becton Dickinson and Co., Sparks, Maryland, USA). The lungs were lavaged twice with PBS $(0.25 \mathrm{ml}$ followed by $0.2 \mathrm{ml}$ ); approximately $0.3 \mathrm{ml}$ of the instilled fluid was consistently recovered. Total cell counts were determined using a hemocytometer. Cell pellets were resuspended in PBS, and smears were prepared by cytocentrifugation (Shandon Inc., Pittsburgh, Pennsylvania, USA) at $10.12 \mathrm{~g}$ for 2 minutes. Hema 3 (Biochemical Sciences Inc., Swedesboro, New Jersey, USA) was used to stain all smears. Differential counts of BAL cells were determined from at least 500 leukocytes using standard hemocytological criteria to classify the cells as neutrophils, eosinophils, lymphocytes, or macrophages/monocytes. Additionally, blood was collected by retro-orbital bleeding. Serum was obtained by centrifugation after incubating whole blood for 30 minutes at $37^{\circ} \mathrm{C}$. Finally, lung tissue was fixed in $10 \%$ formalin and embedded in paraffin. Sections $(3-\mu \mathrm{m}$-thick) were stained with hematoxylin and eosin (H\&E).

Splenocyte culture. Spleens were harvested into sterile tubes containing sterile HBSS (Life Technologies Inc., Burlington, Ontario, Canada). Tissue was triturated between the ends of sterile frosted slides, and the resulting cell suspension was filtered through nylon mesh (BSH Thompson, Scarborough, Ontario, Canada). Red blood cells were lysed with ACK lysis buffer $\left(0.5 \mathrm{M} \mathrm{NH}_{4} \mathrm{Cl}, 10 \mathrm{mM} \mathrm{KHCO}_{3}\right.$, and $0.1 \mathrm{nM} \mathrm{Na}_{2}$ EDTA [pH 7.2-7.4]). Remaining splenocytes were washed twice with HBSS and then resuspended in RPMI supplemented with 10\% FBS (Life Technologies Inc.), $1 \%$ L-glutamine, $1 \%$ penicillin/streptomycin. Cells were
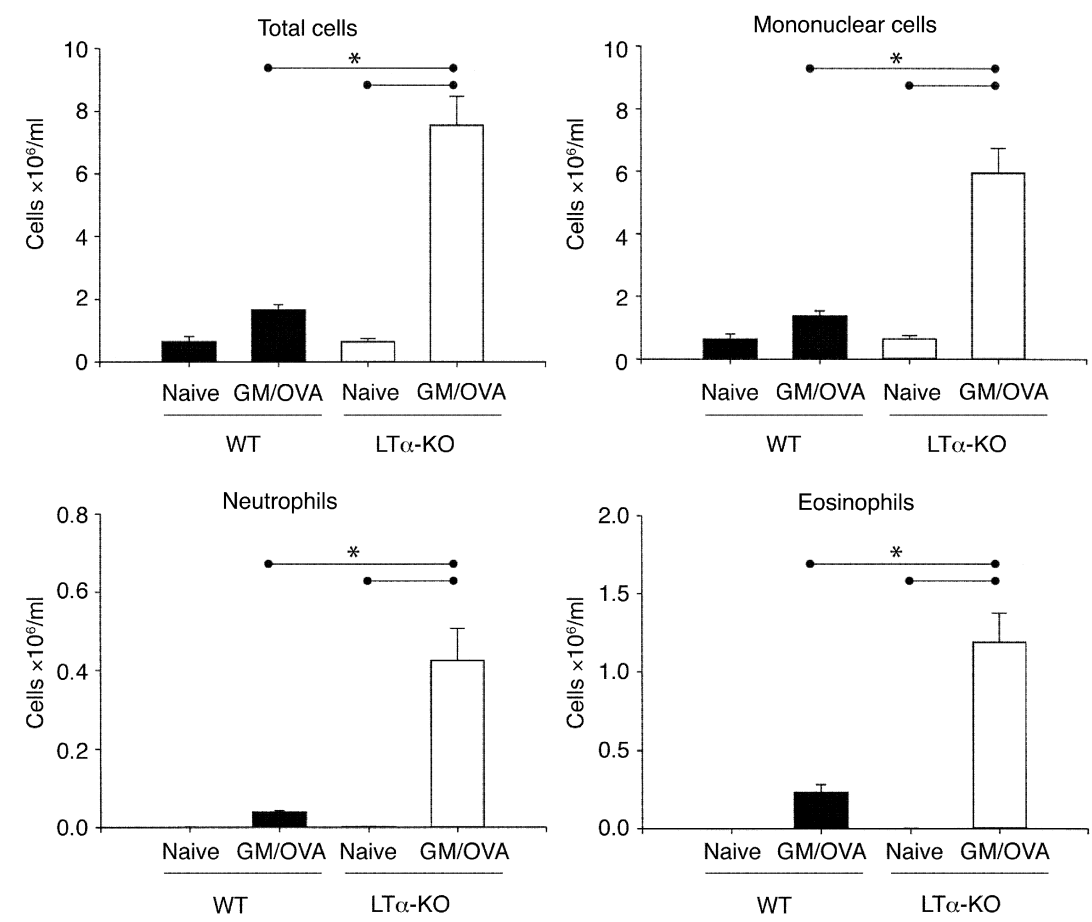

Figure 1

Airway eosinophilia in BAL of C57BL/ 6 (WT) and LT- $\alpha$-deficient ( $L T \alpha-K O$ ) mice exposed to aerosolized OVA in the context of GM-CSF expression. Over a period of 10 consecutive days, mice were exposed daily to OVA. Twenty-four hours before first exposure, mice were infected intranasally with adenoviral construct expressing GM-CSF. Data were obtained 48 hours after last exposure to OVA. (Mean $\pm \mathrm{SEM} ; n=4-6$; statistical analysis was performed using one-way ANOVA with Fisher's post hoc test; ${ }^{*} P<0.05$.) 
cultured in medium alone or with $40 \mu \mathrm{g}$ OVA per well at $8 \times 10^{5}$ cells per well in a flat-bottom, 96-well plate (Becton Dickinson, Franklin Lakes, New Jersey, USA). After 5 days of culture, supernatants were harvested for cytokine measurements.

Cytokine and Ig measurement. ELISA kits for murine IL-13, and IFN- $\gamma$, were purchased from R\&D Systems Inc. (Minneapolis, Minnesota, USA), whereas the kit for murine IL-5 was obtained from Amersham Pharmacia Biotech (Buckinghamshire, United Kingdom). Each of these assays has a threshold of detection of $3-5 \mathrm{pg} / \mathrm{ml}$. Levels of OVA-specific IgE were measured using an antigen-capture ELISA method described previously (11).

Flow cytometric analysis. Flow cytometric analysis was performed on lung cells isolated as described previously with slight modifications (11). Briefly, total lung mononuclear cells were isolated by collagenase digestion (Collagenase type III; Life Technologies Inc., Rockville, Maryland, USA) followed by discontinuing gradient centrifugation in 30\% and 60\% Percoll (Pharmacia Biotech $\mathrm{AB}$, Uppsala, Sweden). Interface containing mononuclear cells was collected, washed twice with PBS, and stained with a panel of antibodies. In addition, we performed flow cytometric analyses on white blood cells. To this end, blood was collected from the abdominal vein into $1-\mathrm{ml}$ syringes coated with heparin. Red blood cells were lysed with lysing buffer $\left(150 \mathrm{mM} \mathrm{NH}_{4} \mathrm{Cl}, 0.1 \mathrm{mM}\right.$ disodium EDTA, and $10 \mathrm{~nm} \mathrm{NaHCO}$ ), and samples were subjected to three washes with PBS. Total white cell number in peripheral blood was counted with a hemocytometer, and cells were stained with appropriate antibodies. The following antibodies were purchased from PharMingen (Mississauga, Ontario, Canada): anti-CD3 (PEconjugated 145-2CII), anti-CD4 (biotin-conjugated L3T4), and anti-MHC class II (FITC-conjugated 25-917). The T1/ST2 (3E10) antibody was provided by Millennium Pharmaceuticals Inc. (Cambridge, Massachusetts, USA), and FITC was labeled in-house according to a standard protocol (12). To minimize nonspecific binding, $10^{6}$ cells were preincubated with FcBlock (CD16/CD32; PharMingen). For each antibody combination, $10^{6}$ cells were incubated with $\mathrm{mAb}$ 's at $0-4^{\circ} \mathrm{C}$ for 30 minutes; the cells were then washed and treated with second-stage reagents. Streptavidin PerCP (Becton Dickinson Immunocytometry Systems, San Jose, California, USA) was used as a second-step reagent for detection of biotin-labeled antibodies. Titration was used to determine the optimal concentration for each antibody. Cells were fixed in $1 \%$ paraformaldehyde and counted on a FACScan (Becton Dickinson and Co.), and analyses were performed using WinMDI software (The Scripps Research Institute, La Jolla, California, USA). A total of 20,000-30,000 events were acquired.

Surgical removal of spleen. Mice were anesthetized with isoflurane, and buprenorphine was administered subcutaneously. Splenectomy was preformed as described previously (13). After surgical skin preparation, the spleen was exteriorized through a $1-\mathrm{cm}$ left subcostal incision. The splenic artery and vein were double ligated and the spleen was removed. The peritoneum and skin were closed in separate layers using 4.0 absorbable suture. Mice were rested for 10 days before commencement of our allergic sensitization protocol.

Data analysis. Data are expressed as mean \pm SEM. Statistical interpretation was performed using ANOVA with Fisher's post hoc test or Student's $t$ test. Differences were considered statistically significant when $P<0.05$.

\section{Results}

Immune-inflammatory responses in the airway. $\mathrm{LT} \alpha-\mathrm{KO}$ mice and WT littermates subjected to our protocol of respiratory mucosal allergic sensitization were sacrificed 48 hours after the last OVA aerosolization, and the BAL cellular profile was assessed (Figure 1). The BAL cellular content in naive mice from either strain was very similar. Compared with naive controls, both strains mounted an inflammatory response after sensitization. The extent of the response in WT mice was similar to that reported previously (11). Interestingly, the inflammatory response detected in the BAL of LT $\alpha-\mathrm{KO}$ mice was approximately five times greater than that observed in sensitized WT mice. Owing to the unequal variance between strains,
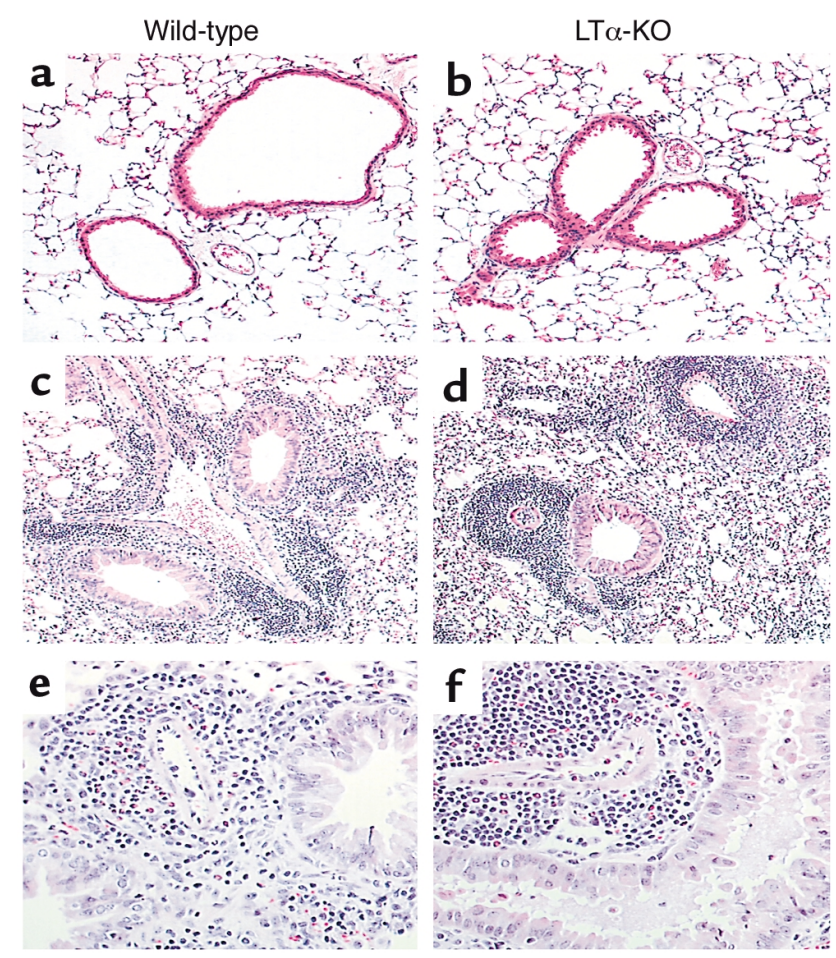

\section{Figure 2}

Light photomicrograph of paraffin-embedded sections of lung tissues. Over a period of 10 consecutive days, mice were exposed to OVA, and tissues were obtained 48 hours after last exposure. Sections were stained with $\mathrm{H} \& \mathrm{E}$. Panels represent naive mice ( $\mathbf{a}$ and $\mathbf{b}$ ) and mice exposed to OVA in the context of GM-CSF expression (c-f). $\times 50$ (a-d); $\times 200$ (e and f). 

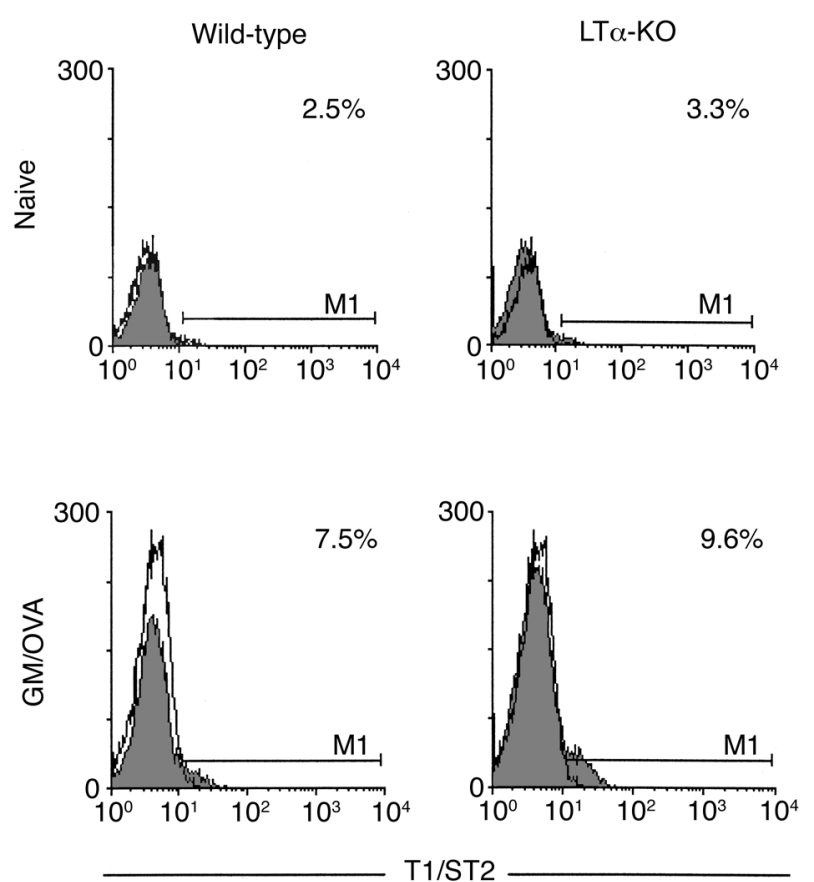

statistical significance between naive and OVA-treated WT mice was not reached, as assessed by one-way ANOVA. However, a $t$ test conducted only on WT mice showed statistical significance. Proportionately, the percentage of eosinophils in LT $\alpha$-KO and WT mice was similar ( $15.5 \pm 2.0 \%$ vs. $13.8 \pm 2.5 \%$, respectively); in contrast, the percentage of lymphocytes in LT $\alpha$-KO mice was considerably greater, $37 \pm 5 \%$ vs. $5 \pm 3 \%$ in WT mice.

Histological examination of lung tissues paralleled the findings observed in the BAL (Figure 2). In agreement with our previous reports, sensitized WT mice showed a marked accumulation of inflammatory cells that was both perivascular and peribronchial; evidence of goblet cell hyperplasia and mucus production was readily apparent. In naive $\mathrm{LT} \alpha-\mathrm{KO}$ mice, there was no indication of peribronchial inflammation, but we observed scattered mild mononuclear perivascular infiltrates. The response to allergic sensitization was qualitatively similar in both strains, with extensive peribronchial inflammation characterized primarily by eosinophils and mononuclear cells. Of note, the perivascular infiltrate of LT $\alpha-\mathrm{KO}$ mice was rather massive and consisted almost exclusively of mononuclear cells.

\section{Figure 4}

Flow cytometric analysis of T cells $\left(\mathrm{CD}^{+} / \mathrm{CD}^{+}\right)$and APC $(\mathrm{MHC} \mathrm{II})$ obtained from lungs of naive C57BL/ 6 and LT $\alpha-K O$. (a) Lung mononuclear cell fraction was obtained by enzymatic digestion of whole lung. Lung cells from three to four mice were pooled for each group. Bars represent data obtained from three independent experiments. (Mean \pm SEM; statistical analysis was performed using Student's $t$ test; ${ }^{*} P<0.05$.) (b) Cells were stained with indicated antibodies or alternatively with isotype controls, and 30,000 events were collected. Data are representative of three independent experiments that yielded similar results.

\section{Figure 3}

T1/ST2 expression on T cells obtained from lung tissues after exposure to OVA in the context of GM-CSF. Lungs were collected 48 hours after last exposure and subjected to enzymatic digestion. Cells were stained with mAb's against CD3, CD4, and T1/ST2 and analyzed by flow cytometry. Histograms were gated on $\mathrm{CD}^{+} / \mathrm{CD} 4^{+} \mathrm{T}$ lymphocytes. Open histograms represent the background staining with the isotype control antibody. Data are representative of two independent experiments that yielded similar results.

To determine whether the considerable infiltration of mononuclear cells reflected an accumulation of Th2 cells, we examined the percentage of $\mathrm{CD}^{+} \mathrm{T}$ cells expressing T1/ST2, a putative marker of Th2 polarized cells (14). As indicated in the histograms shown in Figure 3 , the percentage of $\mathrm{CD}^{+} / \mathrm{CD}^{+}$cells expressing this marker was similar in naive mice of both strains. This percentage increased approximately threefold in both WT and LT $\alpha$-KO mice after OVA sensitization.

Flow cytometric analysis of lung cells from naive $C 57 B L / 6$ and $L T \alpha-K O$ mice. Given that the airway inflammatory response observed in $\mathrm{LT} \alpha-\mathrm{KO}$ mice was considerably greater than that observed in WT controls, we used flow cytometric analysis to investigate aspects of the immunological status of lungs from naive mice of both strains. Figure 4a shows the absolute number of lung

a

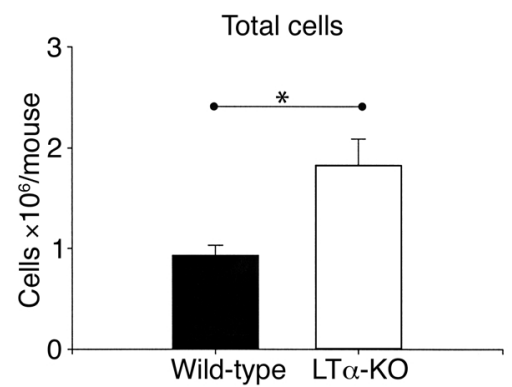

b
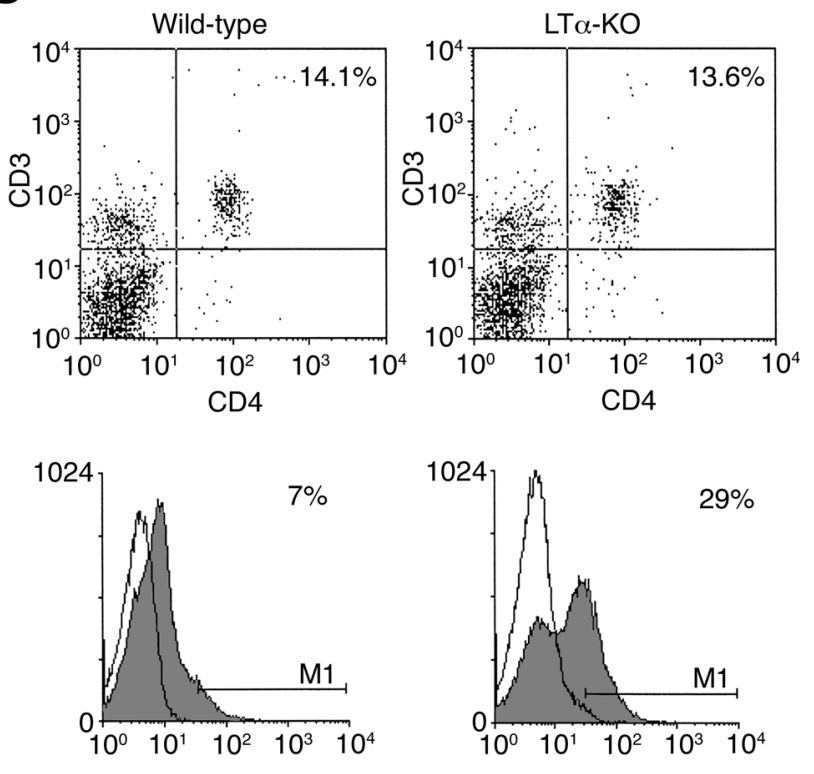

$\mathrm{MHC}$ II 

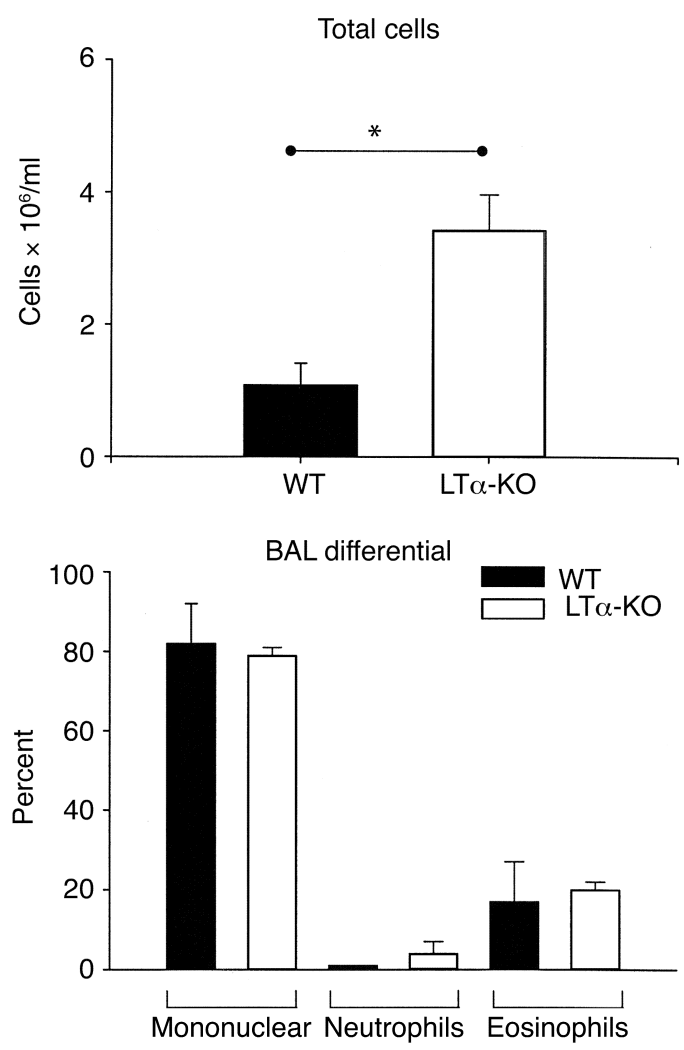

mononuclear cells isolated. In agreement with our histological observations, the number of mononuclear cells in LT $\alpha-\mathrm{KO}$ mice was approximately $40 \%$ greater, although the percentage of $\mathrm{CD}^{+} / \mathrm{CD}^{+}$cells was similar in both strains, at approximately $14 \%$ (Figure $4 \mathrm{~b}$ ). In contrast, the percentage of cells expressing MHC II was greater in LT $\alpha-\mathrm{KO}$ mice (29\%) than in WT mice $(7 \%)$, suggesting that $\mathrm{LT} \alpha-\mathrm{KO}$ mice may have intrinsically enhanced antigen-presenting capability.

Long term in vivo OVA recall responses. Next, we examined the impact of LN deficiency on the generation of longterm antigen-specific memory. After complete resolution of the acute airway inflammatory response, mice were reexposed to OVA (day 60). Seventy-two hours after the last exposure, they were sacrificed and the BAL cellular response assessed. As shown in Figure 5, LT $\alpha$-KO mice mounted a robust mononuclear and eosinophilic airway inflammatory response upon OVA reexposure. Cytokine production of cultured splenocytes and OVA-specific serum IgE. Effector cytokines were measured in the supernatant of splenocyte cultures. This was done for two reasons: first, our experience is that it is generally difficult to detect cytokines in the BAL of mice with a C57BL/6 background in our model; second, it was particularly important in these studies to obtain evidence of systemic Th effector activity. As shown in Table 1, the levels of IFN- $\gamma$, IL-5, and IL-13 detected in the supernatant of unstimulated splenocytes from either strain was very low. Splenocytes from sensitized mice of both strains produced considerably greater levels of these three cytokines. This response was three to four times greater in $\mathrm{LT} \alpha-\mathrm{KO}$ mice.

\section{Figure 5}

In vivo rechallenge of C57BL/ 6 (WT) and LT- $\alpha$-deficient ( $L T \alpha-K O)$ mice exposed to OVA in the context of GM-CSF. At day 60 , after complete resolution of the airway inflammation, mice were reexposed to aerosolized OVA for 3 consecutive days. Data show total cell number and differential of cells obtained 72 hours after last challenge from BAL (mean $\pm \mathrm{SEM} ; n=4-6$; statistical analysis was performed using Student's $t$ test; ${ }^{*} P<0.05$ ).

Table 1 provides additional evidence with respect to levels of OVA-specific $\operatorname{IgE}$ in serum, indicating that the response was quantitatively similar in WT as in $\mathrm{LT} \alpha-\mathrm{KO}$ mice subjected to the protocol of allergic sensitization. Impact of splenectomy in the generation of allergic sensitization. We addressed the role of the spleen in the generation of allergic airway inflammation. To this end, we surgically removed spleens of both WT and LT $\alpha-\mathrm{KO}$ mice and, after full recovery, subjected the mice to the respiratory mucosal allergic sensitization protocol. The airway inflammatory response in splenectomized WT mice was not significantly different from that observed in eusplenic sensitized control mice (Figure 6a). In contrast, splenectomized LT $\alpha$-KO mice were unable to generate an airway inflammatory response. In fact, total and differential cell counts in the BAL were identical to those of naive LT $\alpha-\mathrm{KO}$ mice (Figure $6 \mathrm{~b}$ ). The histological evaluation of lungs from these mice agreed with the BAL data (Figure 6c).

Peripheral blood changes before and after splenectomy. To assess the impact of splenectomy on number and distribution of white blood cells (specifically $\mathrm{T}$ cells and $\mathrm{B}$ cells) in the peripheral blood, blood samples were collected before and 10 days after surgical removal of the spleen and subjected to flow cytometric analyses. Total white blood cell counts were greater in $\mathrm{LT} \alpha-\mathrm{KO}$ than in WT mice preceding and following splenectomy (Table 2). However, removal of the spleen did not result in significant changes in cell numbers in either WT or LT $\alpha$ $\mathrm{KO}$ mice. In addition, although splenectomy in WT mice did not have an impact on $\mathrm{T}$ and $\mathrm{B}$ cells distribution, we observed an increase in both $\mathrm{CD}^{+} / \mathrm{CD}^{+}$and $\mathrm{CD}^{+} / \mathrm{CD}^{+}$cells in $\mathrm{LT} \alpha-\mathrm{KO}$ mice.

\section{Table 1}

Antigen-specific cytokine and IgE production

\begin{tabular}{lcccc} 
& \multicolumn{2}{c}{ WT } & \multicolumn{2}{c}{ KO } \\
& Medium & OVA & Medium & OVA \\
IFN- $\gamma$ & $14 \pm 5$ & $2732 \pm 777$ & $10 \pm 2$ & $9978 \pm 4967$ \\
IL-5 & $0 \pm 0$ & $82 \pm 44$ & $0 \pm 0$ & $363 \pm 121$ \\
IL-13 & $129 \pm 19$ & $1928 \pm 541$ & $95 \pm 32$ & $3757 \pm 1037$ \\
& Naive & GM/OVA & Naive & GM/OVA \\
IgE & $2 \pm 0.5$ & $176 \pm 61$ & $0 \pm 0$ & $127 \pm 32$
\end{tabular}

For cytokine measurement, splenocytes were obtained from C57BL/6 (WT) or LT $\alpha-K O(K O)$ mice after nine OVA exposures. Cells were cultured for 5 days in medium alone or stimulated with OVA. Data represent picograms per milliliter, mean $\pm \mathrm{SEM} ; n=4$ per group. Levels of OVA-specific IgE were measured in serum of WT and KO mice obtained 48 hours after last OVA exposure. Data represent units per milliliter, mean $\pm \mathrm{SEM} ; n=4$ per group. 

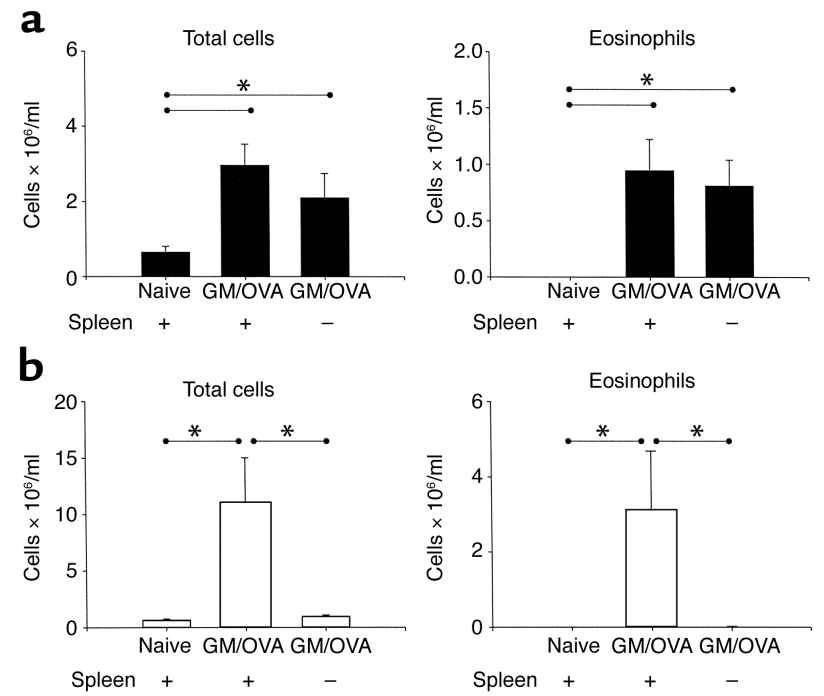

C

Wild-type
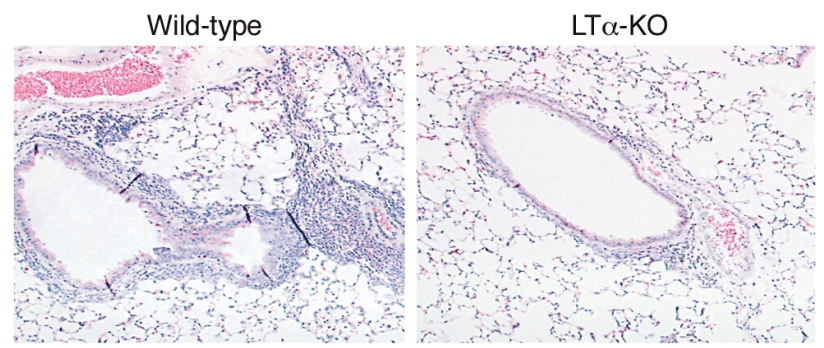

\section{Discussion}

To our knowledge, this is the first demonstration of intact antigen-specific Th2 immunity upon mucosal sensitization of LT $\alpha-\mathrm{KO}$ mice. Indeed, compared with WT mice, LT $\alpha$-KO mice responded to OVA aerosolization in the context of GM-CSF with a similar pattern of T1/ST2 expression on Th2 cells and a robust Th2-associated inflammatory response characterized primarily by eosinophilia in the lung tissue. Moreover, after resolution of the initial response, OVA reexposure readily reconstituted airway eosinophilic inflammation, indicating antigen specificity and the persistence of immunological memory. As further evidence of Th2 immunity, LT $\alpha$-KO mice were able to generate OVAspecific IgE responses. This is of particular interest in the light of previous data indicating the compromised ability of $\mathrm{LT} \alpha-\mathrm{KO}$ mice to generate antibody responses against KLH, HSV-1, and SRBC $(9,15)$. It is also of interest because germinal centers, which are considered to be essential for Ig isotype switching, are absent in these mutant mice. However, Matsumoto et al. (16) recently demonstrated near-normal affinity maturation and isotype switching in $\mathrm{LT} \alpha-\mathrm{KO}$ mice after repeated challenge with high doses of antigen (NP-OVA) together with an adjuvant. As suggested by Wang et al. (17) and illustrated by our experimental system, persistence of antigen in the context of adjuvant plausibly explains Ig affinity maturation of $\mathrm{B}$ cells outside germinal centers.

The magnitude of the airway inflammatory response in LT $\alpha$-KO mice exposed to aerosolized OVA in the con-

\section{Figure 6}

Impact of splenectomy on the generation of inflammation in airways of $\mathrm{C} 57 \mathrm{BL} / 6$ and $\mathrm{LT} \alpha-\mathrm{KO}$ mice. Mice were splenectomized as described in Methods. Ten days after surgery, mice were infected intranasally with $\mathrm{Ad} / \mathrm{GM}-\mathrm{CSF}$ and subsequently exposed to OVA. BAL samples were obtained 48 hours after last OVA exposure from C57BL/ 6 (a) and LT $\alpha$ $\mathrm{KO}$ (b) mice. (Mean $\pm \mathrm{SEM} ; n=4$; statistical analysis was performed using ANOVA with Fisher's post hoc test; ${ }^{*} P<0.05$.) (c) Light photomicrograph of lung sections stained with $\mathrm{H} \& \mathrm{E} . \times 50$.

text of GM-CSF was rather striking. Indeed, whether in terms of total number of cells or of differential cell counts, the degree of the inflammatory response in the mutant mice was four- to sixfold greater than that observed in WT controls. As documented histologically (Figure 2), and in agreement with Banks et al. (9), part of the reason may be that there is an increased number of mononuclear cells in the perivascular areas of naive mutant mice. However, this difference (Figure 4a) appears quantitatively insufficient to explain the changes documented after exposure to our allergic sensitization protocol. A contributing factor may be that, as a result of lacking lymphoid structures (other than the spleen), there is an increased number of immune cells circulating in LT $\alpha-\mathrm{KO}$ mice, as described previously by De Togni et al. (8) and confirmed in our studies (Table 2). We provide evidence of an additional, potentially important mechanism underlying the enhanced immune responsiveness observed in the mutant mice: a dramatic increase in the number of $\mathrm{MHCII}^{+}$cells in the lung of naive mice: $29 \%$ in $\mathrm{LT} \alpha-\mathrm{KO}$ versus $7 \%$ in WT mice. That $\mathrm{LT} \alpha-\mathrm{KO}$ mice have an increased capacity for presenting antigen may also explain the very recent observation by Lee et al. (18) of enhanced immune responses of $\mathrm{LT} \alpha-\mathrm{KO}$ mice to murine gammaherpes virus 68 .

Compelled by the observation that mice lacking LN were able to generate Th2 immunity and airway eosinophilic inflammation, we investigated whether the spleen, albeit architecturally aberrant in LT $\alpha-\mathrm{KO}$ mice, was able to compensate as the site of the primary immune response. The ability of splenocytes from LT $\alpha-$ KO mice subjected to repeated OVA aerosolizations in the context of GM-CSF to produce cytokines upon OVA recall in vitro argues for the role of the spleen in this

\section{Table 2}

Changes in peripheral blood lymphocytes following splenectomy

\begin{tabular}{ccccc}
\hline & $\begin{array}{c}\text { WBC } \\
\left(\text { cells } \times 10^{6} / \mathrm{ml}\right)\end{array}$ & $\begin{array}{c}\mathrm{CD}^{+} / \mathrm{CD}^{+} \\
(\%)\end{array}$ & $\begin{array}{c}\mathrm{CD}^{+} / \mathrm{CD}^{+} \\
(\%)\end{array}$ & $\begin{array}{c}\mathrm{B}^{220^{+}} \\
(\%)\end{array}$ \\
$\mathrm{WT} /+\mathrm{SP}$ & $5.8 \pm 1.6$ & $12.9 \pm 1.5$ & $6.7 \pm 1.6$ & $70.5 \pm 3.3$ \\
$\mathrm{~W} /-\mathrm{SP}$ & $8.6 \pm 2.9$ & $20.8 \pm 2.3$ & $10.1 \pm 1.5$ & $63.1 \pm 5.5$ \\
$\mathrm{~K} /+\mathrm{SP}$ & $33.2 \pm 8.8^{\mathrm{A}}$ & $17.5 \pm 1.1$ & $7.1 \pm 0.6$ & $73.2 \pm 2.0$ \\
$\mathrm{~K} /-\mathrm{SP}$ & $24.7 \pm 3.6^{\mathrm{B}}$ & $27.7 \pm 0.1^{\mathrm{C}}$ & $17.8 \pm 1.4^{\mathrm{C}}$ & $59.8 \pm 4.8$
\end{tabular}

Changes in peripheral blood were collected from naive C57BL/6 (WT) and $\mathrm{LT} \alpha-\mathrm{KO}(\mathrm{KO})$ mice before (+SP) or after (-SP) splenectomy as described in Methods. Cells were stained with antibodies against indicated markers and subjected to flow cytometric analysis. A gate was set up for lymphocytes and 30,000 events were collected. ${ }^{\text {AS }}$ tatistically different from WT/+SP. ${ }^{B}$ Statistically different from WT/-SP. CStatistically different from $\mathrm{KO} /+\mathrm{SP}$. 
process. That cytokine content in splenocyte cultures supernatant was, in fact, two to three times greater in LT $\alpha-\mathrm{KO}$ mice than in WT control mice suggests an immune response of greater strength in the mutant mice. A decisive functional role for the spleen in allergic sensitization was provided by the splenectomy experiments. Indeed, splenectomy of LT $\alpha$-KO mice fully prevented the development of airway inflammation. Our data are different from those of Davis et al. (13), who demonstrated that removal of the spleen in LT $\alpha-\mathrm{KO}$ mice decreased, but did not abrogate, humoral responses to Salmonella in intestines. In our experiments, mice were subjected to allergic sensitization protocol 10 days after splenectomy; at this time, the peripheral cell counts of $\mathrm{LT} \alpha-\mathrm{KO}$ mice before and after splenectomy were not significantly different, indicating that the absence of sensitization and airway inflammation was not due to depletion of circulatory leukocytes and, specifically, of T cells $\left(\mathrm{CD}^{+} / \mathrm{CD}^{+}\right.$ and $\mathrm{CD}^{+} / \mathrm{CD}^{+}$) or $\mathrm{B}$ cells (Table 2). Although these data demonstrate that the spleen indeed supplanted the role of the regional LN in LT $\alpha$-KO mice, splenectomy of WT mice had no impact on the ability of asplenic mice to generate allergic sensitization and airway inflammation.

Our current understanding of the generation of mucosal immunity is that antigens penetrating mucosae are captured by DCs and transported to the draining LNs to initiate immune responses (7). The data presented here indicate that the spleen can facilitate allergic sensitization to antigens introduced into the respiratory mucosae. This observation evokes an important question: How does aerosolized OVA reach the spleen to initiates allergic immunity? Wolvers et al. (5) have demonstrated that after OVA delivery to the nasal mucosae, there is evidence of APCs transporting this antigen to the spleen. This DC migration may occur via the bloodstream or, presumably, through the lymphatic system, which, importantly, remains intact in LT $\alpha-\mathrm{KO}$ mice. In our experimental system, OVA aerosolization leads to eosinophilic airway inflammation only in the context of GM-CSF transgene expression. Such expression is compartmentalized within the lung/airway, as GM-CSF cannot be detected in the circulation. Therefore, we surmise that it is very unlikely that exogenous GM-CSF expression in the spleen contributed to the ability of $\mathrm{LT} \alpha-\mathrm{KO}$ mice to generate allergic sensitization. Thus, our data intimate that the generation of allergic priming in the spleen of mice lacking LNs may be the result of mobilization of OVA-carrying DC from the respiratory mucosae.

In short, our data show that allergic sensitization and airway inflammation take place in the absence of thoracic (and other) LNs. That these responses are abrogated in splenectomized mice establishes the essential requirement of secondary lymphoid organs and implies that the lung does not possess the intrinsic capability to generate allergic sensitization. That the spleen can supplant the role of the draining LNs illustrates the plasticity of the immune system and highlights the systemic nature of allergic sensitization. What is the potential clinical significance of these findings? The current emphasis of the pharmacological management of asthma rests on local treatment, which effectively inhibits inflammatory processes that take place in the airway itself. However, one would argue that processes that are generated, and probably maintained, outside the airway will likely evade this treatment strategy. Thus, attempts to ultimately cure, rather than control, allergic airway inflammation may need to incorporate a systemic dimension.

\section{Acknowledgments}

This research has been supported by grants from the Canadian Institutes of Health Research (CIHR). We also acknowledge the support of the Hamilton Health Science Corp. and the St. Joseph Hospital Foundation. B.U. Gajewska and D. Alvarez hold CIHR doctoral fellowships. M.R. Stämpfli holds a Parker B. Francis Fellowship. We are grateful to Monika Cwiartka for technical support, Mary Kiriakopoulos for secretarial assistance, and Ryan E. Wiley for critical review of the manuscript.

1. Banchereau, J., and Steinman, R.M. 1994. Dendritic cells and control of immunity. Nature. 392:245-252.

2. Lambrecht, B.N., et al. 2000. Myeloid dendritic cells induce Th2 responses to inhaled antigen leading to eosinophilic airway inflammation. $J$. Clin. Invest. 106:551-559.

3. Lambrecht, B.N., Pauwels, R.A., and Fazekas De St. Groth, B. 2000. Induction of rapid $\mathrm{T}$ cell activation, division, and recirculation by intratracheal injection of dendritic cells in a TCR transgenic model. $J$. Immunol. 164:2937-2946.

4. Xia, W., Pinto, C.E., and Kradin, R.L. 1995. The antigen-presenting activities of $\mathrm{Ia}^{+}$dendritic cells shift dynamically from lung to lymph node after an airway challenge with soluble antigen. J. Exp. Med. 181:1275-1283.

5. Wolvers, D.D., et al. 1999. Intranasally induced immunological tolerance is determined by characteristics of the draining lymph nodes: studies with OVA and human cartilage gp-39. J. Immunol. 162:1994-1998.

6. Hoyne, G.F., Askonas, B.A., Hetzel, C., Thomas, W.R., and Lamb, J.R. 1996. Regulation of house dust mite responses by intranasally administered peptide: transient activation of CD4+ T cells precedes the development of tolerance in vivo. Int. Immunol. 8:335-342.

7. Vermaelen, K.Y., Carro-Muino, I., Lambrecht, B.N., and Pauwels, R.A. 2001. Specific migratory dendritic cells rapidly transport antigen from the airways to the thoracic lymph nodes. J. Exp. Med. 193:51-60.

8. De Togni, P., et al. 1994. Abnormal development of peripheral lymphoid organs in mice deficient in lymphotoxin. Science. 264:703-707.

9. Fu, Y.X., et al. 1997. Lymphotoxin-alpha (LTalpha) supports development of splenic follicular structure that is required for IgG responses. J. Exp. Med. 185:2111-2120.

10. Ruddle, N.H. 1999. Lymphoid neo-organogenesis: lymphotoxin's role in inflammation and development. Immunol. Res. 19:119-125.

11. Stämpfli, M.R., et al. 1998. GM-CSF transgene expression in the airway allows aerosolized ovalbumin to induce allergic sensitization in mice. J. Clin. Invest. 102:704-714.

12. Golding, J.W. 1976. Conjugation of antibodies with fluorochromes: modification of the standard method. J. Immunol. Methods. 13:215-219.

13. Davis, I.A., Knight, K.A., and Rouse, B.T. 1998. The spleen and organized lymph nodes are not essential for the development of gut-induced mucosal immune responses in lymphotoxin-alpha deficient mice. Clin. Immunol. Immunopathol. 89:150-159.

14. Lohning, M., et al. 1998. T1/ST2 is preferentially expressed on murine Th2 cells, independent of interleukin 4 , interleukin 5 , and interleukin 10, and important for Th2 effector function. Proc. Natl. Acad. Sci. USA. 95:6930-6935.

15. Banks, T.A., et al. 1995. Lymphotoxin-alpha-deficient mice. Effects on secondary lymphoid organ development and humoral immune responsiveness. J. Immunol. 155:1685-1693.

16. Matsumoto, M., et al. 1996. Affinity maturation without germinal centres in lymphotoxin-alpha-deficient mice. Nature. 382:462-466.

17. Wang, Y., et al. 2000. Antigen persistence is required for somatic mutation and affinity maturation of immunoglobulin. Eur. J. Immunol. 30:2226-2234

18. Lee, B.J., Santee, S., Von Gejsen, S., Ware, C.F., and Sarawar, S.R. 2000 Lymphotoxin-alpha-deficient mice can clear a productive infection with murine gammaherpesvirus 68 but fail to develop splenomegaly or lymphocytosis. J. Virol. 74:2786-2792. 